\title{
Rotational mixing and Lithium depletion
}

\author{
M. H. Pinsonneault ${ }^{1}$ \\ ${ }^{1}$ Ohio State University, Dept. of Astronomy 140 W. 18th Ave. Columbus, OH 43210 USA \\ email: pinsonneault.1@osu.edu
}

\begin{abstract}
I review basic observational features in Population I stars which strongly implicate rotation as a mixing agent; these include dispersion at fixed temperature in coeval populations and main sequence lithium depletion for a range of masses at a rate which decays with time. New developments related to the possible suppression of mixing at late ages, close binary mergers and their lithium signature, and an alternate origin for dispersion in young cool stars tied to radius anomalies observed in active young stars are discussed. I highlight uncertainties in models of Population II lithium depletion and dispersion related to the treatment of angular momentum loss. Finally, the origins of rotation are tied to conditions in the pre-main sequence, and there is thus some evidence that environment and planet formation could impact stellar rotational properties. This may be related to recent observational evidence for cluster to cluster variations in lithium depletion and a connection between the presence of planets and stellar lithium depletion.
\end{abstract}

Keywords. Hydrodynamics - stars: abundances, rotation, spots

\section{Introduction}

Lithium is an extraordinarily sensitive diagnostic of stellar structure and evolution. The observed lithium abundances in stars, not surprisingly, reveal an extremely complex picture, and it can sometimes be difficult to remember why rotational mixing is a useful framework for interpreting this data. I therefore begin by briefly summarizing the case for rotation as the physical ingredient responsible for light element depletion in stars.

\subsection{Evidence for rotational mixing}

The first and most important point is that stellar rotation is capable of driving mild envelope mixing at the observationally required rates (Pinsonneault et al. 1989.) Rotation induces a departure from spherical symmetry which generates meridional circulation currents, and both structural evolution and angular momentum loss from magnetized winds generate shears which can drive mild turbulence. Lithium is easily destroyed in stellar interiors, and such mild mixing can therefore generate surface lithium depletion.

This leads directly to a second important feature of rotational mixing which is observationally required: namely, stars which rotate at different rates will have different mixing histories. Rapid rotators experience stronger torques and larger shears than slow rotators, and they also are less spherical. It is therefore a basic prediction of rotational mixing that there should be a dispersion in mixing rates which can manifest itself as a dispersion in lithium at fixed mass, composition, and age. Lithium is observed to have a significant dispersion in many clusters (see Pinsonneault 1997 for a theoretical review and Sestito \& Randich 2005 for a more recent observational synthesis) while other elements in open clusters are very uniform (Paulson et al. 2003). Other mechanisms, such as gravity waves and microscopic diffusion, can generate depletion but not dispersion, so this observed feature allows us to discriminate between physical processes. 
Finally, both the mass dependence and time dependence of the observed depletion pattern strongly implicate rotationally driven mixing as the culprit. Rotation declines with age, and so does lithium depletion. By contrast, processes such as gravitational settling tend to be more independent of age, or even increase in rate as stars get older. Rotational mixing also extends through stellar envelopes, and as a result it can simultaneously mix different elements and be present in stars with very different surface convection zone depths. We observe lithium depletion in all low mass open cluster stars, which would not be expected if lithium depletion were a phenomenon confined to the convection zone boundary. This does not rule out interesting interactions with other physics processes, such as magnetic or wave-driven angular momentum transport (see the contribution by Talon in these proceedings), but it does require rotation as a component of the solution.

However, the physics of stellar angular momentum evolution is extremely challenging, and it has proven difficult to develop a rigorous physical model. This has led to a sort of stasis in our understanding of phenomena such as rotational mixing. Fortunately, there have been positive developments, which I summarize below, which reveal a dynamic and more complete picture of stellar evolution. In Section 2 recent advances in our understanding of angular momentum evolution are reviewed; Section 3 then discusses three areas where there are either new observational or theoretical features in stellar lithium depletion. A discussion of some recent developments is given in section 4 .

\section{Angular momentum evolution}

Stellar rotation is an initial value problem, and the initial conditions are set by the details of the star formation process. The angular momentum distribution is subsequently modified by angular momentum loss (via star-disk interactions) and internal angular momentum transport. The physics of the latter is vigorously debated in the literature, with three distinct mechanisms (hydrodynamic, wave-driven, and magnetic) all being in principle important. Rotational mixing is a natural byproduct of angular momentum transport in stellar radiative interiors, especially from hydrodynamic mechanisms. This is a rich field, so I will summarize the main developments relevant for rotational mixing.

Stars appear at the deuterium-burning birthline (Stahler 1988) with a range of rotation rates, typically well below that expected for accretion from a Keplerian disk. The currently favored explanation is that magnetic coupling between the protostar and the accretion disk regulates the rotation (Shu et al. 1994.) In this framework, the initial rotation rate can be thought of as related to the mass accretion rate in the early hydrodynamic stages of star formation. However, the predicted rotation rates on the main sequence are both too rapid and too uniform if models with the observed rotation rates are evolved to the main sequence, even if torques from solar-like winds are included.

However, if a coupling between protostars and their accretion disks exists, the initial spread of rotation rates can be amplified and stars can reach the main sequence as relatively slow rotators. Much observational work has also been invested in the question of star-disk coupling, with a diversity of results largely centered around the proper choice of disk proxies and disentangling evolutionary effects. However, recent Spitzer studies (Rebull et al. 2006) have provided strong evidence for a relationship between rotation and the presence of disks. This may reflect a coupling between the protostar and accretion disk similar to that operating at the earlier stages, or it could be induced by an enhanced stellar wind tied to accretion. In either case, the lifetime of accretion disks and their degree of coupling to the parent star is crucial for establishing the main sequence rotation. Rotation is therefore now perceived as a product of environment, and this raises the 
interesting possibility that rotational mixing may also depend on where a star was born or on how the accretion disk evolved.

There are also now very large databases of stellar rotation periods, ranging from star forming regions (Rodriguez-Ledesma et al. 2009) to extensive open cluster surveys such as the Monitor program(Irwin et al. 2009) and transit studies such as the one which yielded a large database of rotation periods in the 550 Myr system M37(Hartman et al. 2009). The latter study in particular indicates the ability of modern campaigns to infer rotation periods caused by spot modulation for large stellar samples at small amplitude.

These samples can in turn be used to reconstruct the angular momentum evolution of stellar populations, in particular the dependence of angular momentum loss on rotation rate and mass, as well as the coupling timescale between core and envelope (e.g. Irwin et al. 2007, Denissenkov et al. 2009). Different groups agree on the essential features. Angular momentum loss scales as the rotation rate cubed at low rotation rates, then saturates at a threshold which decreases as mass decreases. The net effect is that lower mass stars take longer to spin down and longer for their rotation rates to converge. The cores of the slowly rotating population couple to their envelopes with a timescale of order $100 \mathrm{Myr}$, while rapidly rotating stars appear to be more strongly coupled. These results are consistent with helioseismic data indicating that the rotation of the solar interior is strongly coupled to that of the surface convection zone.

This combination of theoretical advances and improved rotational data and empirical constraints therefore has significant promise for more robust rotational mixing predictions in the future.

\section{Lithium depletion and rotational mixing revisited}

The basic rotational mixing picture can be simply defined. Stars experience a massdependent pre-main sequence lithium burning epoch, which ends when they develop substantial radiative cores. They then experience rotational mixing on the main sequence, induced either by shears generated by angular momentum loss or their departure from spherical symmetry. As the stars spin down the rate of lithium depletion decreases. This overall picture is reasonable, but a number of phenomena defy easy categorization within it. This is in large part because of the interaction of rotation with other phenomena typically neglected in stellar models. Below are three examples.

\subsection{Structural effects of starspots}

There is a striking dispersion in lithium abundances among late-type stars in young open clusters; the Pleiades is the clearest example (Soderblom et al. 1993.) This trend is not expected from rotational mixing in such young stars, and the relative effect is also the opposite of the one expected: namely, the least depleted stars are the most active and heavily spotted. Much subsequent work has focused on whether the dispersion is real or induced by the heavily spotted nature of the stars in question; the model atmospheres used to interpret the data typically neglect the large changes in the strength of the lithium feature which would be associated with a substantial fraction of the surface covered with cool spots with ample neutral lithium. However, in recent work (King et al. 2009) we found that the scatter in K I was much less than the scatter in Li I, indicating that the bulk of the dispersion is real. The likely origin in our view is actually a different mechanism altogether, and it is motivated by recent data on radius anomalies in active stars from interferometric and eclipsing binary studies.

Eclipsing binaries such as YY Gem (Torres \& Ribas 2002) were found to have radii significantly larger than those predicted by interiors theory. Subsequent work traced out 
a radius anomaly pattern. More recent interferometric data permits the measurement of radii for inactive field stars, which are found to be in accord with theoretical predictions (Demory et al. 2009.) High activity, such as that found in tidally synchronized short-period binaries, therefore appears to puff up stars. A similar effect during the fully convective protostellar phase would reduce the degree of pre-main sequence lithium depletion; if this varied from star to star it could generate a dispersion with characteristics remarkably like the data. This is illustrated in Fig. 1., where Pleiades data from Soderblom et al. (1993) is compared with standard stellar models (lower line) and models with a radius inflated by 10 percent, the level inferred in highly active stars (upper line). In addition to being an attractive solution for a longstanding problem, this leads to an interesting insight. Stellar activity is not a mere detail; it can impact the entire structure of a star and change its mixing history.

\subsection{Blue stragglers and halo Lithium depletion}

The lithium depletion pattern in metal-poor stars poses a different problem; the majority of stars exhibit little dispersion and the observed abundances appear to be nearly independent of surface temperature or metallicity. One striking counter-example is the presence of a small but real population of highly depleted stars (Thorburn 1994.) In recent work on blue stragglers we find that a population of sub-turnoff merger products, presumably highly lithiumn depleted, is predicted to arise from such mergers; the number expected is close to that observed in halo stars (Andronov et al. 2006). This confirms the suggestion in Ryan et al. (2001) that these highly depleted stars should not be regarded as the tail of a rotational mixing distribution, but rather that they have a distinct origin.

This does not, however, require that mixing be absent in halo stars. The nature of the dispersion predicted depends on both the distribution of initial conditions and the angular momentum loss history. At present we can only extrapolate Population I conditions to Population II stars. Their torques and initial conditions could very well have been different; for example, planet formation may be less common in them, and this could impact the distribution of accretion disk coupling timescales (see Section 4.) Future work on the activity properties of tidally synchronized halo stars may prove diagnostics of the braking law, while we may need rotation data in more metal-poor outer disk systems to test the metallicity dependence of the initial conditions.

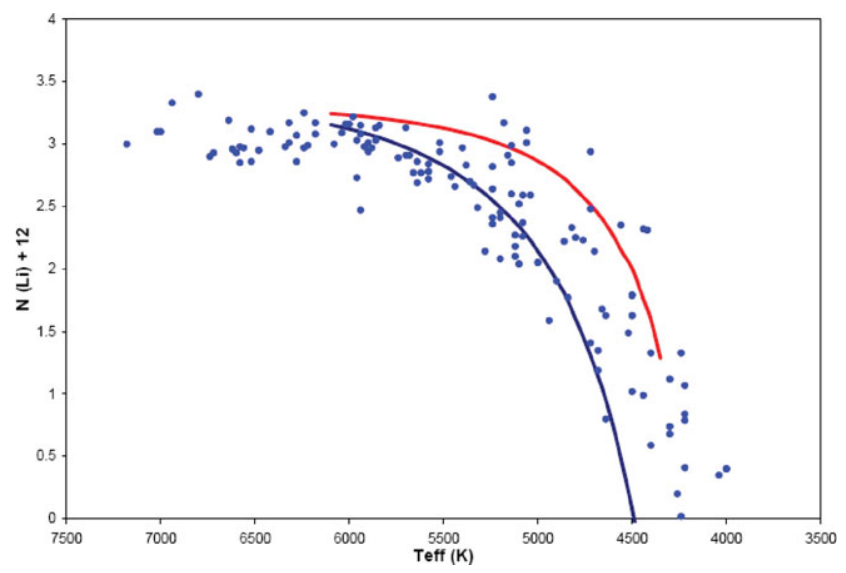

Figure 1. Lithium abundances as a function of effective temperature in the Pleiades cluster compared with standard models (lower line) and models which were inflated during the pre-main sequence lithium depletion epoch (upper line). 


\subsection{Interaction of diffusion and rotational mixing}

Microscopic diffusion (or gravitational settling and thermal diffusion) is a basic physical process expected to occur in stars, typically over a very long timescale. The net effect is that heavy species tend to sink relative to light ones, although radiation pressure can drive some heavier elements upwards in sufficiently thin surface convection zones (Michaud 1970). There is clear evidence for diffusion in the Sun (Bahcall \& Pinsonneault 1992), both in the sound speed profile and in the detection of a surface helium abundance lower than that initially required to reproduce the solar luminosity.

Diffusion can induce lithium depletion directly, but it also has interesting interactions with rotational mixing (see Richard et al. 1996 for a nice example in the solar context). Gravitational settling operates over shorter timescales for thinner convection zones, and it produces mean molecular weight gradients at the base of the surface convection zone. It is energetically unfavorfable to mix in the presence of a mu gradient, and mixing can erase composition gradients; there is thus a natural competition between the two processes. Furthermore, the timescale for mixing increases with age, while the timescale for settling changes very slowly (and tends to decrease as stars evolve to higher effective temperatures). One might therefore expect rotational mixing to predominate earlier while diffusion suppresses mixing at later ages, and for this interaction to depend on mass and composition. This may be related to the apparent stalling of lithium depletion in older open clusters (discussed by Randich in these proceedings), and could be an additional source of lithium depletion in halo stars as well. Recent evidence for settling in multiple elements (Korn, these proceedings) of globular cluster stars provides evidence that diffusion sets in for older stars; an earlier epoch of depletion is certainly permitted by theory, although establishing this observationally will require additional work as discussed in the angular momentum evolution section. Observations of multiple elements, as already done in globulars, could be used to establish the diffusion signature in old open clusters and the interaction between mixing and separation.

\section{Future directions}

In closing I'd like to note some other wrinkles which may prove important for understanding lithium depletion: differences in depletion patterns from cluster to cluster (see the presentation by Randich) and an apparent excess lithium depletion in stars which host planets (see the talks by Israelian and, for a contrary view, Melendez.) Both can be interpreted in the framework where stellar rotation properties are determined by interactions between protostars and accretion disks. In dense stellar environments the timescale for interactions can be comparable to the lifetimes inferred for accretion disks, raising the possibility that stars born in such regions might have a different distribution of disk lifetimes than stars born in loose associations. This hypothesis is testable in the measured rotation rates of young systems, and this is an important potential effect (especially if we use clusters as an evolutionary sequence!) which needs to be explored.

The recent report that stars with planets have excess lithium depletion (Israelian et al. 2009) may be a fascinating example of how the formation of planets can impact the properties of stars. Bouvier (2008) has proposed a linkage, arguing that systems with planets should have long-lived accretion disks. These in turn become slow rotators, with large relative shears, which in turn could drive excess mixing. He thus argued that there may be a connection between lithium overdepletion and planet formation. Such a link is certainly plausible, but the opposite correlation appears to be required by rotational mixing. More rapid rotators experience larger absolute torques (and in 
any case subsequently evolve to become slow rotators, thus in effect adding the mixing from the rapid to that of the slow phase). They also experience larger departures from spherical symmetry; both imply stronger mixing. However, the rotation is set primarily by the coupling between star and disk, not necessarily in the disk lifetime itself, and this may explain the apparent contradiction between "massive disk required for planets" and "weak star-disk interaction required for rapid rotation and lithium depletion." This avenue may prove promising to explore, and it would be a delightful turn of events if the planetary tail could wag the stellar dog.

\section{References}

Andronov, N., Pinsonneault, M. H., \& Terndrup, D. M. 2006, ApJ, 646, 1160

Bahcall, J. N. \& Pinsonneault, M. H. 1992, RMP, 64, 885

Bouvier, J. 2008, AEA A (Letters), 489, 53

Demory, B.-O., Ségransan, D., Forveille, T., Queloz, D., Peuzit, J. L., et al. 2009, A\& A, 505, 205

Denissenkov, P., A., Pinsonneault, M. H., Terndrup, D. M., \& Newsham, G. 2009, submitted ApJ (astro-ph/0911.1121)

Hartman, J. D., Gaudi, B. S., Pinsonneault, M. H., Stanek, K. Z., Holman, M. J., McLeod, B. A., Meibom, S., Barranco, J. A., \& Kalirai, J. S. 2009, ApJ, 691, 342

Irwin, J., Hodgkin, S., Aigrain, S., Hebb, L., Bouvier, J., Clarke, C., Moraux, E., \& Bramich, D. M., 2007, MNRAS, 377, 741

Irwin, J., Aigrain, S., Bouvier, J., Hebb, L., Hodgkin, S., Irwein, M., \& Moraux, E. 2009, MNRAS, 392, 1456

Israelian, G., Delgado Mena, E., Santos, N. C., Sousa, S. G., Mayor, M., Udry, S., Dominguez Cerdena, C., Rebolo, R., \& Randich, S. 2009, Nature, 462, 189

King, J. R., Schuler, S. C., Hobbs, L. M., \& Pinsonneault, M. H. 2009, ApJ in press (astro$\mathrm{ph} / 1001.2796)$

Michaud, G. 1970, ApJ, 160, 641

Paulson, D. B., Sneden, C., \& Cochran, W. D. 2003, AJ, 125, 3185

Pinsonneault, M. H. 1997, ARAA, 35, 557

Pinsonneault, M. H., Kawaler, S. D., Sofia, S., \& Demarque, P. 1989, ApJ, 338, 424

Rebull, L. M., Stauffer, J. R., Megeath, S. T., Hora, J. L. \& Hartmann, L. 2006, ApJ, 646, 297

Richard, O., Vauclair, S., Charbonnel, C., \& Dziembowski, W. A.. 1996, A\&A, 312, 1000

Rodriguez-Ledesma, M. V., Mundt, R., \& Eisloffel, J. 2009 A $\&$ A, 502, 883

Ryan, S. G., Beers, T. C., Kajino, T., \& Rosolankova, K. 2001, ApJ, 547, 231

Sestito, P. \& Randich, S. 2005, A\&\&A, 442, 615

Shu, F. H., Najita, J., Ostriker, E., Wilkin, F., Ruden, S., \& Lizano, S. 1994, ApJ, 429, 781

Soderblom, D., Jones, B. F., Balachandran, S., Stauffer, J. R., Duncan, D. K., Fedele, S. B., \& Hudon, J. D. 1993, AJ, 106, 1059

Stahler, S. W. 1988, ApJ, 332, 804

Thorburn, J. A. 1994, ApJ, 421, 318

Torres, G. \& Ribas, I. 2002, ApJ, 567, 1140 